\title{
Structure and functions of Orange Carotenoid Protein homologs in cyanobacteria
}

Han Bao ${ }^{a}$, Matthew R. Melnicki, ${ }^{a, b}$, Cheryl A. Kerfeld ${ }^{a, b, c, d, *}$

a MSU-DOE Plant Research Laboratory, Michigan State University, East Lansing, MI 48824, USA

${ }^{\mathrm{b}}$ Molecular Biophysics and Integrated Bioimaging Division, Lawrence Berkeley National Laboratory, Berkeley, CA 94720, USA

${ }^{\mathrm{C}}$ Department of Biochemistry and Molecular Biology, Michigan State University, East Lansing, MI 48824, USA

d Department of Plant and Microbial Biology, University of California, Berkeley, CA 94720, USA

*Address correspondence to: ckerfeld@lbl.gov

The author responsible for distribution of materials integral to the findings presented in this article is: Cheryl A. Kerfeld (ckerfeld@lbl.gov).

Address: MSU-Department of Energy Plant Research Laboratory, Michigan State University, East Lansing, Michigan 48824

Phone: 1-(517)-432-4371

E-mail:

Han Bao hanbao@msu.edu

Matthew R. Melnicki_mmelnicki@lbl.gov 


\section{Abstract}

Rapidly-induced photoprotection in cyanobacteria involves thermal dissipation of excess energy absorbed by the phycobilisome (PBS), the primary light-harvesting antenna. This process is called non-photochemical quenching (NPQ), and is mediated by a water-soluble photoactive protein, the Orange Carotenoid Protein (OCP). The OCP is structurally and functionally modular, consisting of a sensor domain, an effector domain, and a carotenoid. Blue-green light induces a structural transition of the OCP from the orange inactive form, $\mathrm{OCP}^{O}$, to the red active form, $O C P^{R}$. Translocation of the carotenoid into the effector domain accompanies photoactivation. The $\mathrm{OCP}^{\mathrm{R}}$ binds to the PBS core, where it triggers dissipation of excitation energy and quenches fluorescence. To recover the antenna capacity under low light conditions, the Fluorescence Recovery Protein (FRP) detaches the OCP from the PBS and accelerates back-conversion of $O C P^{R}$ to $O C P^{O}$. Increased sequencing of cyanobacterial genomes has allowed the identification of new paralogous families of the OCP and its domain homologs, the Helical Carotenoid Proteins (HCPs), which have been found distributed widely among taxonomically and ecophysiologically diverse cyanobacteria. Distinct functions from the canonical OCP have been revealed for some of these paralogs by recent structural and functional studies. 


\section{Introduction}

Photoprotective mechanisms have evolved in photosynthetic organisms to cope with fluctuating light and environmental stresses. Regulation of these mechanisms is critical for maintaining photosynthetic efficiency by minimizing photodamage. Typically, excess excitation energy that cannot be used for photochemistry in photosystem II (PSII) is dissipated as heat. This process is called non-photochemical quenching (NPQ) [1]. The mechanisms of NPQ differ among cyanobacteria, algae and plants. In contrast to the relatively well-characterized systems operational in eukaryotes [2], the Orange Carotenoid Protein (OCP)-mediated photoprotective response of cyanobacteria has only been extensively studied in the last decade $[3,4]$.

The OCP is a water-soluble, $35 \mathrm{kDa}$ protein that binds a single carotenoid molecule $[3,4]$. It is the only known photoactive protein that uses a carotenoid as its chromophore. The absorption of blue-green light or strong white light causes the OCP to convert from a dark stable orange form, $O C P^{O}$, to a light activated red form, $O C P^{R}$. The $O C P^{R}$ form directly participates in photoprotection by binding to the light-harvesting antenna, the phycobilisome (PBS; Figure 1). Recovery from the energy-dissipative state is catalyzed by the Fluorescence Recovery Protein (FRP). The photoprotective OCP-FRP regulatory system (Figure 1 ) is found in the majority of cyanobacteria (with the exception of Prochlorococcus and marine Synechococcus) (Figure 2).

In addition to high light, expression of the OCP is known to be upregulated also under various stress conditions (e.g. nutrient starvation, oxidative stress, salt stress [4]). In addition, the OCP was proposed to govern the selective energy flow from the antenna to the different photosystems [5] in megacomplexes consisting of both photosystems plus the PBS, suggesting another means by which the OCP could play a role in acclimation to stress conditions at the level of the photosystems. The OCP is also known to quench singlet oxygen $\left({ }^{1} \mathrm{O}_{2}\right)[6,7]$ and has also been proposed to play a role in transport of carotenoids to the cytoplasmic or outer membrane to form a photoprotective shield [8]; this implies that the OCP also functions as part of systemic subcellular stress responses. Recent bioinformatic, structural, functional, and biophysical studies have 
revealed numerous unanticipated findings, including new families of OCP (Bao et al., manuscript in revision) and OCP domain homologs $[9,10]$ which confer an expanded range of functions. Collectively, an evolutionary history of the OCP is emerging which suggests that this group of soluble carotenoproteins has been of fundamental importance to the early evolution of cyanobacteria and perhaps even for the development of oxygenic photosynthesis.

\section{Structural and Functional Modularity of the OCP System}

Long before its precise function was understood, the crystal structure of $\mathrm{OCP}^{\circ}$ [6] revealed that it is comprised of two discrete structural domains: an all $\alpha$-helical $\mathrm{N}$ terminal domain (NTD) unique to cyanobacteria, and a mixed $\alpha / \beta C$-terminal domain (CTD) that shares structural similarity to other proteins within the widespread nuclear transport factor 2 (NTF2)-like superfamily known to bind substrates with keto or hydroxyl functionalized 6 -carbon rings $[6,11]$. The two domains are connected by a flexible linker and by a non-covalently bound ketocarotenoid molecule which is embedded within a tunnel-like binding pocket that spans both domains (Figure 3A). The OCP can bind different carotenoids, however the presence of a keto group is essential for photoactivity [12-14]. The carbonyl group of ketocarotenoids (e.g. 3'-hydroxyechinenone, echinenone, or canthaxanthin) forms two hydrogen bonds with residues in the CTD (Y201 and W288) that are absolutely conserved [12,15]. These hydrogen bonds are essential for photoactivity; OCP containing zeaxanthin, which lacks the carbonyl group, abolishes photoactivity [16]. The $\mathrm{OCP}^{\mathrm{O}}$ consistently crystallizes as a dimer that, based on structural data (e.g. buried surface area, $\Delta G$ of association) is proposed to be a physiologically-relevant form, however whether OCP is a monomer or a dimer is controversial $[6,11,17,18]$. Interestingly, the two carotenoids within the OCP dimer are aligned nearly parallel to one another $[6,8]$, which may have biological significance.

Using controlled proteolysis experiments, it was demonstrated that the OCP's structural modularity extends to function [19]: in the absence of the CTD, the NTD of the OCP retains the carotenoid, is spectrally similar to $O C P^{R}$, and constitutively quenches phycobilisome fluorescence in vitro - the hallmark feature of OCP activity. NTDs formed by proteolysis, Red Carotenoid Proteins (RCPs) [19,20], have been isolated from 
cyanobacteria [21]. The CTD appears to regulate the fluorescence quenching/photoprotective role of the NTD by priming carotenoid photochemistry in $\mathrm{OCP}^{\mathrm{O}}$ and by acting as a substrate for $\mathrm{FRP}$ in $\mathrm{OCP}^{\mathrm{R}}$. This structural and functional modularity is reminiscent of other bacterial photoreceptor that are composed of discrete sensor and effector domains [22].

The RCP consists of the NTD with a bound carotenoid [19,20]. Relative to its position in the $\mathrm{OCP}^{\mathrm{O}}$ structure, the carotenoid is shifted $12 \AA$ to be enveloped by the NTD. The position of the carotenoid in $O C P^{R}$ is shown to correspond to that in $\operatorname{RCP}[18,20]$ (Figure 3B). Although the crystal structure of the $O C P^{R}$ is not yet available, various biochemical and biophysical methods [15,17,18,23-28], such as small-angle X-ray scattering (SAXS) [18], X-ray hydroxyl radical footprinting [18,23,25], mass spectrometry (MS) $[17,25,29]$, and circular dichroism [18] have suggested that the OCP undergoes a significant global conformational change upon photoactivation, with complete separation of the NTD and CTD [18,23] (Figure 1A). Thus, the OCP undergoes a light-driven domain dissociation, predicated upon an "unbolting" of the carotenoid which normally contributes to domain association in the $\mathrm{OCP}^{\circ}$ form [30].

With the advent of whole genome sequencing, Wu and Krogmann [21] first identified that the slr1963 gene in Synechocystis corresponded to the OCP, and this gene was consistently accompanied by a second open reading frame [8] that is now known to be the FRP [31,32]. The Synechocystis FRP crystallized as two forms, a dimer and an elongated tetramer [33] (Figure 3C). The dimeric form is the active form which interacts with the CTD of the OCP [33-35]; the tetramer is likely an inactive, potentially storage form of the protein [33].

\section{Mechanism of OCP Quenching}

In plants, NPQ occurs at the membrane-embedded light-harvesting chlorophyll antenna complexes (LHCs) [1,2], which can switch from a light-harvesting state to a photoprotective state through protonation-induced conformational changes in LHC proteins with consequent dissipation of surplus excitation energy. An decrease in lumen $\mathrm{pH}$ induces a dimer-to-monomer transition of PsbS protein $[36,37]$ or a conformational change associated with altered lumenal intermolecular interactions of the PsbS dimer 
[38]. This activates the xanthophyll cycle, whereby a chemical interconversion by enzymatic de-epoxidation of violaxanthin occurs under high light conditions and can lead to accumulation of zeaxanthin, which is an effective quencher of excited chlorophyll molecules and also facilitates the aggregation of LHCs, which can subsequently decouple from PSII in plants.

In contrast to $\mathrm{pH}$-triggered $\mathrm{NPQ}$ in plants, the OCP-mediated quenching mechanism in cyanobacteria is simpler [39]. Illumination with blue-green light (or strong white light) triggers OCP-mediated quenching at the water-soluble PBS antenna complexes in the cytoplasm. Instead of changing the carotenoid by chemical reactions, as in the xanthophyll cycle of plants, cyanobacteria alter carotenoid properties by shifting its protein environment within the OCP as a result of photoactivation, stabilizing it in a more planar conformation that enhances its effective conjugation length [14]. Concomitant changes in the interaction between the CTD and the NTD include the breakage of a conserved salt bridge - Arg155-Glu244 - at the major interface between the two domains and detachment of the N-terminal extension (NTE) from the CTD at the minor interface, allowing separation of the two domains (Figure 1A); the unwinding of the NTE helix [18] is the only detectable change in secondary structure upon photoactivation.

The NTD in the open, elongated $O C P^{R}$ form attaches to the PBS and brings the carotenoid to the PBS chromophores to induce energy quenching. The $O C P^{R}$ form is stabilized upon binding to the PBS [39], which may be influenced by interaction between the positively charged Arg-155 accessible on the newly exposed NTD surface and negative surface charges in the PBS core [40].

The PBS binding site where the OCP/RCP docks is a topic of active investigation $[17,41,42]$. Recently, the basal cylinders of the allophycocyanin (APC) core were proposed to be the site of $O C P^{R}$ binding $[41,43,44]$. These APC trimers include multiple $\mathrm{APC}_{660}$ species and at least two $\mathrm{APC}_{680}$ species, with an $\mathrm{APC}_{660}$ bilin suggested to be the primary site of fluorescence quenching according to in vivo and in vitro picosecond fluorescence measurements [45,46]. Involvement of an $\mathrm{APC}_{680}$ bilin has also been proposed as the location of OCP-mediated quenching [47], implicating the terminallyemitting large core-membrane linker, $A p c E$, also known as $L_{C M}[41,42,48]$. Blue-light- 
inducible NPQ reminiscent of OCP behavior has been observed even in the primitive cyanobacterium Gloeobacter violaceus PCC 7421, which has atypical PBS structure and lacks thylakoid membranes [49]. Although the PBS rods and the rod-core linker proteins are different between $G$. violaceus and model OCP organisms like Synechocystis, the structure of their PBS cores are very similar, which supports that quenching occurs at or near the APC trimers.

In the mechanism of OCP-mediated NPQ, it is possible that the OCP ${ }^{R}$ binds in close proximity to an excited $\alpha$ - or $\beta$-bilin pigment located in an APC trimer of the PBS core and quenches its excited state directly. An energy transfer [50] or a charge transfer [45] from the bilin pigment to the carotenoid in $O C P^{R}$ has been proposed $[41,45,50-53]$ for de-exciting the antenna. The light-driven translocation of the carotenoid deeper into the NTD [20] could be essential for these mechanisms since this movement could bring the carotenoid closer to the bilins. More speculatively, the carotenoid may undergo an additional translocation - into the core of the PBS - upon binding [20]. Another possibility is that $O C P^{R}$ activates quenching indirectly through a structural modification of a core APC cylinder upon binding; such a structural change could induce a conformational rearrangement of the bilin pigment and thereby interfere with its energy propagation [54].

Chemical cross-linking of the $O C P^{R}$ bound to PBSs revealed several crosslinks to the interdomain linker; only one cross-link identified to the CTD (linking ApcB to CTD residue K249), suggesting that the CTD might not directly interact with the PBS [17,41]. Surprisingly, PBS cross-links were not detected with any NTD residues [41] despite strong evidence that NTD binds PBS on its own [19]; however, strong and specific interaction between the PBS and NTD [40] could have precluded access to the crosslinking agents. When the NTD of $O C P^{R}$ binds to the PBS core, the CTD may be freely mobile (via the interdomain linker), providing accessibility for the FRP to bind and turn off quenching by accelerating the detachment of $O C P^{R}$ from the PBS, thereby enabling its conversion back to $O C P^{\circ}[31,33,39,40]$. An ability to switch off the NPQ state would be very important for avoiding the wasteful quenching of excitation energy under low light conditions. As the activity of FRP favors the accumulation of excitation energy in 
the antenna - and therefore the "fluorescence recovery" for which it is named - it is expected that the FRP would function transiently, as required by changing environmental conditions. However, it currently remains unknown if FRP is able to sense environmental conditions that may regulate its activity.

\section{Multiplicity of OCPs, FRP and Domain Homologs}

Since the first OCP structural description [6] and the earliest sequencing of cyanobacterial genomes, the individual OCP domains were recognized to also be encoded as independent polypeptides [8]. At least nine different clades of homologs to the NTD can now be phylogenomically identified across diverse cyanobacteria (Figure 4A), with members of different clades often co-occurring in the same genome [9] (Figure 2). These paralogs have been named "Helical Carotenoid Proteins" (HCPs), as they are all predicted to conserve both the all-helical structure as well as the carotenoidbinding pocket of the OCP-NTD $[9,10]$. C-terminal domain homologs (CTDHs) which are phylogenetically basal to the OCP-CTD sequences (Figure 4B) are also found in nearly every genome encoding an HCP, although they do not appear to have duplicate copies and their function remains enigmatic [9].

The expansion of the HCP family into different phylogenetic subtypes (Figure 4A) implies that the paralogs may have evolved divergent functions. Expression and characterization of four different subtypes from Nostoc PCC 7120 has shown each to be red-colored like the OCP-NTD and RCP, confirming their ability to bind carotenoids (Figure 4D) $[9,10]$. Functionally, $\mathrm{HCP} 2$ and $\mathrm{HCP} 3$ exhibited strong ${ }^{1} \mathrm{O}_{2}$ quenching activity similar to the OCP-NTD, while HCP4 (containing canthaxanthin) was demonstrated to bind PBSs and enable constitutive energy quenching [10]. A specific function has not yet been demonstrated for HCP1, although crystal structures with different carotenoids suggest its ability to bind a variety of different carotenoids, including myxoxanthophyll which contains a terminal bulky glycosyl group [9]. Therefore, it appears that a wider range of carotenoids bound to HCPs might be able to mediate ${ }^{1} \mathrm{O}_{2}$ quenching, compared to the more specific binding of ketocarotenoids that is required for inducible antenna quenching by the OCP $[6,7]$. Furthermore, the binding of HCP1 with myxoxanthophyll led to altered optical properties compared to HCP1 bound 
with canthaxanthin (Figure 4D). This ability of the HCP/NTD family to bind different types of carotenoids implicates the chromophore as another module which may be varied in order to tune the functional properties of proteins which contain this domain.

In light of the interaction between the NTD and CTD of the OCP, it seems plausible that CTDHs may also interact with some HCPs, forming a heterodimer which would resemble the OCP without its linker (Figure 4C). The CTDH gene locus is commonly adjacent to an HCP4 or HCP5 locus [9], which are the clades which branch closest to the primitive OCP-NTD sequences (Figure 4A) and include the Nostoc 7120 paralog (All4941, an HCP4) which was the only HCP to demonstrate PBS quenching [10]. This evidence suggests that an ancient gene fusion event between an HCP and a CTDH may have occurred, giving rise to an ancestral OCP [9].

At least two additional families of full-length OCP paralogs were also recently identified (Figure 4A, B), occurring in diverse cyanobacterial genomes across most phylogenetic sub-clades (Bao et al., manuscript in revision). One of these new families, OCP2, forms a distinct clade that maps between the primitive "OCPX" family and the canonical, wellcharacterized OCP1 family, suggesting an intermediate evolutionary history. Using Fremyella diplosiphon, a model chromatic-adapting cyanobacterium which contains both OCP1 and OCP2, the properties of these paralogs were compared. The OCP2 was able to quench PBS, however with substantially different properties relative to OCP1; OCP2 showed faster photodynamics, inability to stably accumulate in the active $O C P^{R}$ form at room temperature, absence of dimerization, and lack of FRP interaction. Relative to OCP2, OCP1 has multiple levels of fine regulatory control, enabling flexible access to a wider range of quenching activity, and is thus more evolutionarily refined than the newly identified OCP2 and OCPX. This is consistent with the domain fusion hypothesis and justifies the prevalence of OCP1 among the majority of cyanobacteria. 


\section{Conclusions and Prospects}

Like many photoreceptors, the OCP is structurally and functionally modular. The two structural domains of the OCP and the noncovalently-bound carotenoid each contribute distinct functions which are regulated based on a reversible cycle of carotenoid photoconversion/translocation, light-driven domain dissociation, and inactivation by domain re-association, which may be further enhanced by interaction with the FRP. The widespread presence of domain homologs encoded as separate polypeptides supports their independent origins as HCPs and CTDHs which were likely involved in an ancient domain fusion event. The phylogenetic diversification of HCPs and of full-length OCPs further supports the functional modularity of OCP structural components; the emerging evidence of divergent functional properties among paralogs or while binding different carotenoids presents an opportunity to examine how structure-function relationships can evolve in pigment-binding proteins, with particular relevance to the early evolution of oxygenic photosynthesis and the concomitant need for photoprotective mechanisms.

Understanding of the structural features which influence properties such as the strength of domain association, tuning of optical properties, photoconversion dynamics, and interactions with FRP or PBS will enable OCP-based strategies to become part of the synthetic biology toolkit, which relies on modular components that can be swapped interchangeably. Modular photoreceptor components have already revolutionized the field of optogenetics $[55,56]$.

Furthermore, advances in understanding how the protein environment can govern properties such as carotenoid selectivity and singlet oxygen quenching could enable the engineering of "smart" photoprotection systems - in plants and in cyanobacteria - in a manner comparable to how photosynthetic productivity was improved in plants by acceleration of recovery from photoprotection, thereby avoiding competition between photoprotection and photochemistry [57]. Engineering photoprotection in cyanobacteria could be a means of improving their performance as microbial cell factories. The regulation of OCP has been identified as one target to engineer increased performance and/or efficiency of production strains [58]. At the same time, a comprehensive 
understanding of the natural distribution of OCPs/HCPs/CTDHs in relation to environmental metadata is expected to provide fundamental information on how cyanobacteria adapt to occupy a wide range of ecophysiological niches [4]. 


\section{Acknowledgements}

This work was supported by the National Science Foundation (IOS 1557324). The authors dedicate this manuscript to David W. Krogmann (1931-2016) who discovered the OCP and was a profoundly important mentor to Cheryl A. Kerfeld. 
A
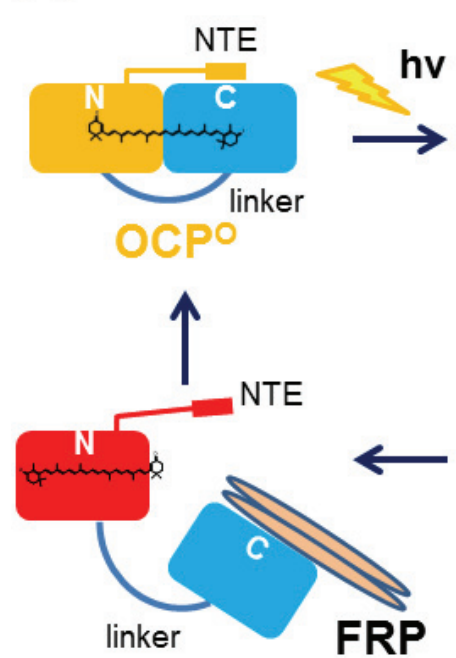
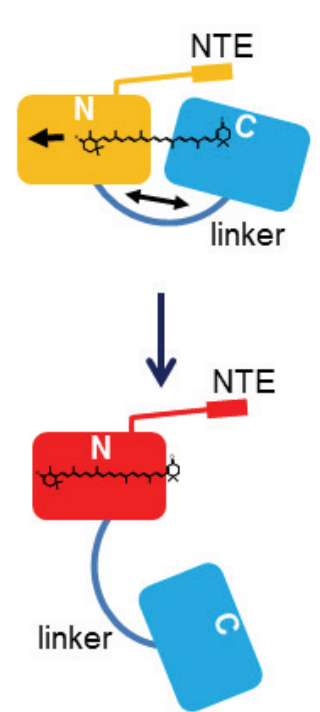

OCPR

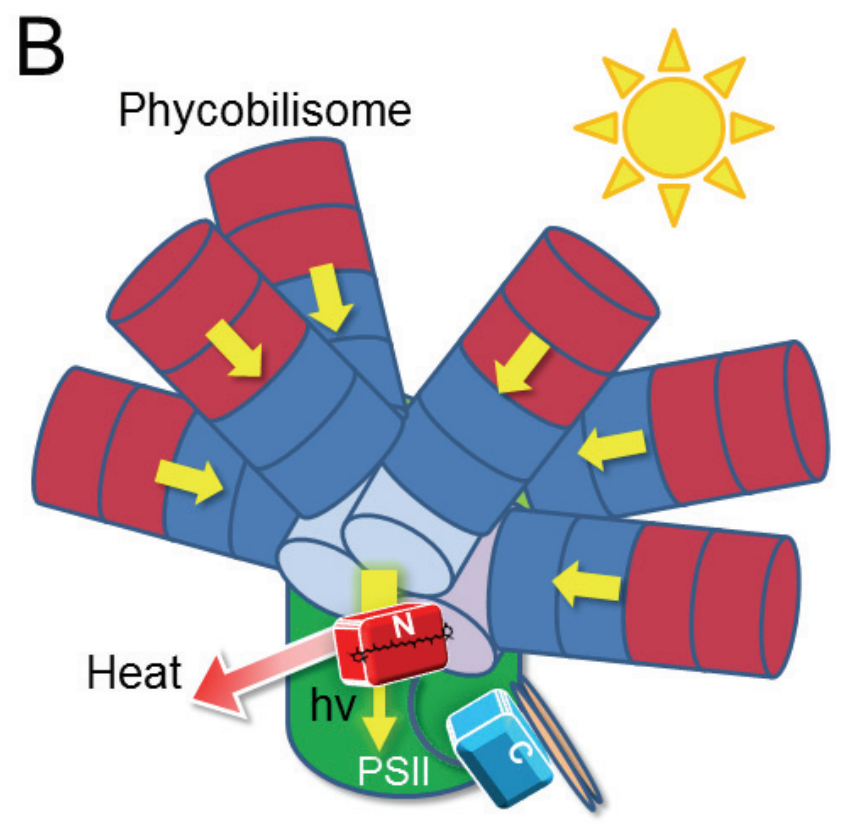

Figure 1. Model of the OCP photocycle and the OCP/FRP-related photoprotective mechanism. A) Mechanism for OCP photoregulation: light absorption by $\mathrm{OCP}^{\mathrm{O}}$ induces conformational changes in the carotenoid and domain interfaces, allowing the carotenoid translocation entirely into the NTD, accompanied by complete dissociation of the two domains. Photoactivated $O C P^{R}$ reverts to inactive $O C P^{O}$ in darkness and can be accelerated by the interaction between the FRP and CTD. B) OCP ${ }^{R}$ interacts with the PBS through the APC trimer of the core to mediate excess energy dissipation as heat. The FRP accelerates the detachment of $O C P^{R}$ from the PBS. 


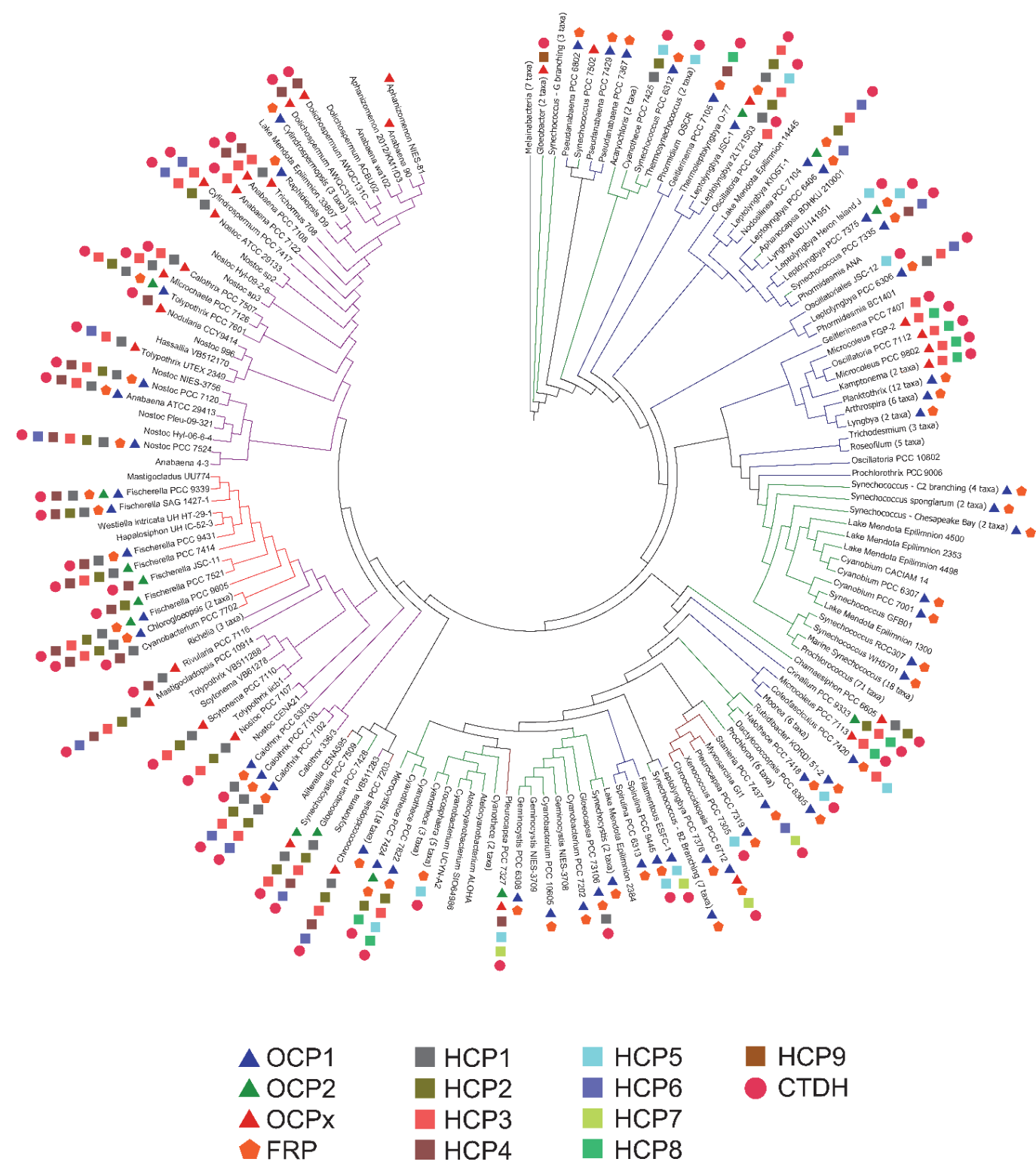

Figure 2. Phylogenetic tree of sequenced cyanobacterial genomes with distribution of OCP-related genes. Presence of genes encoding the OCP, N- and Cterminal domain homologs (HCPs and CTDHs), and the FRP within genomes, denoted as colored shapes, was mapped on a species phylogeny of all Cyanobacteria based on the core housekeeping protein RpoC1 (Bao et al., manuscript submitted). Closely related taxa with the same inventory of OCP-related genes were collapsed, as indicated by the branch names. The five morphological subsections are indicated by the branch color: green (I, unicellular), brown (II, baeocystous), blue (III, filamentous), purple (IV, 
heterocystous)

and

red

(V,

ramified). 

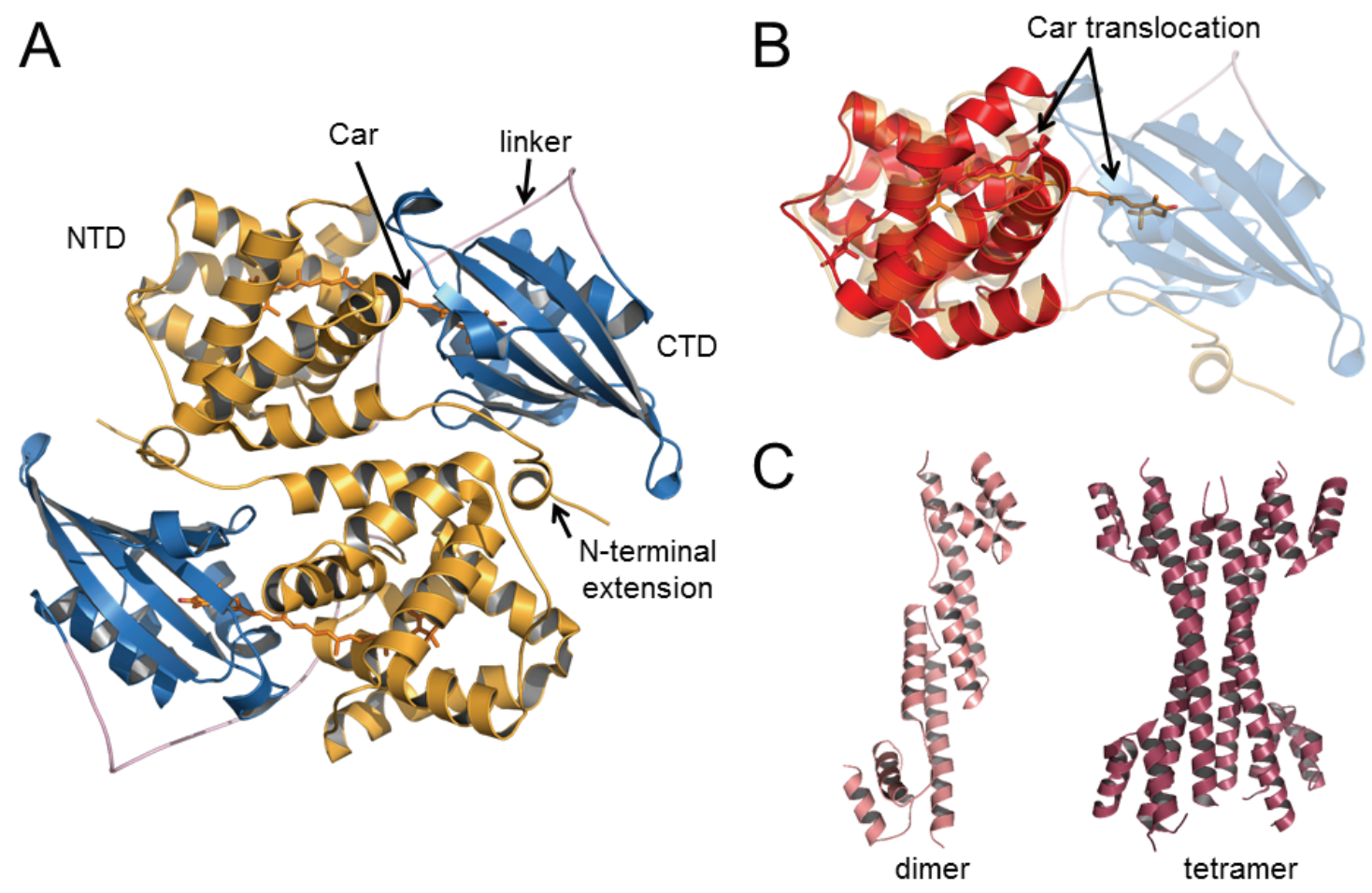

Figure 3. Crystal structures of the $O C P^{\circ}$, RCP and FRP. A) $O C P^{\circ}$ dimer: NTD colored in yellow and CTD colored in blue; the linker colored in pink; the carotenoid (Car) is shown as orange sticks. B) Comparison of the RCP and $\mathrm{OCP}^{\circ}$ structures: RCP (red) aligned with NTD of $\mathrm{OCP}^{\circ}$ (yellow); the carotenoids in the RCP and $\mathrm{OCP}^{\circ}$ are shown by red and orange sticks, respectively, to illustrate the translocation of the carotenoid upon conversion from $\mathrm{OCP}^{\mathrm{O}}$ to $\mathrm{OCP}^{\mathrm{R}}$. C) Two conformations and oligomeric states of the FRP: the active dimeric form shown on the left, and tetrameric form shown on the right. Figure prepared with Pymol (www.pymol.org) using PDB code 4XB5 for OCP, 4XB4 for RCP and 4JDX for FRP. 


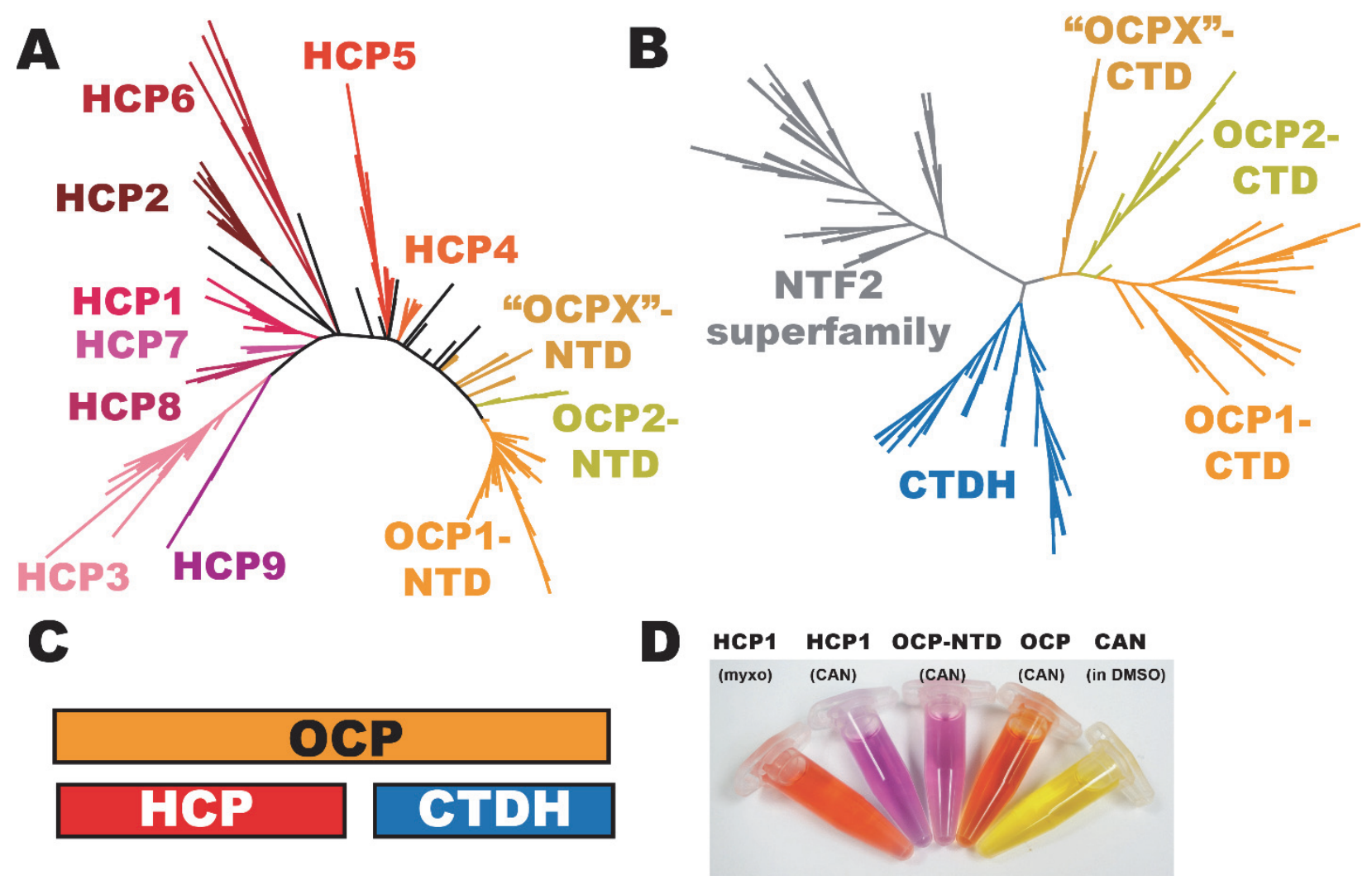

Figure 4. Diversity of domain homologs of the OCP: HCPs and CTDHs. A) ML phylogeny of HCPs and the OCP NTD. B) NJ phylogeny of the OCP-CTD and NTF2 family. C) The HCPs and CTDH are homologous to each structural and functional domain of the OCP. D) Distinctive differences among the visible properties of carotenoproteins. From left to right: Nostoc HCP1 with myxothanthophyll, Nostoc HCP1 with canthaxanthin (CAN), the N-terminal domain of the Synechocystis OCP with CAN, Nostoc OCP with CAN, and free CAN. 


\section{References and recommended reading}

Papers of particular interest, published within the period of review, have been highlighted as:

- of special interest

$\bullet$ of outstanding interest

1. Niyogi KK, Truong TB: Evolution of flexible non-photochemical quenching mechanisms that regulate light harvesting in oxygenic photosynthesis. Curr Opin Plant Biol 2013, 16:307-314.

2. Derks A, Schaven K, Bruce D: Diverse mechanisms for photoprotection in photosynthesis. Dynamic regulation of photosystem II excitation in response to rapid environmental change. Biochim Biophys Acta 2015, 1847:468-485.

3. Kirilovsky D, Kerfeld CA: Cyanobacterial photoprotection by the orange carotenoid protein. Nat Plants 2016, 2:16180.

4. Kirilovsky D, Kerfeld CA: The Orange Carotenoid Protein: a blue-green light photoactive protein. Photochem Photobiol Sci 2013, 12:1135-1143.

5. Liu H, Zhang H, Niedzwiedzki DM, Prado M, He G, Gross ML, Blankenship RE: Phycobilisomes supply excitations to both photosystems in a megacomplex in cyanobacteria. Science 2013, 342:1104-1107.

6. - Kerfeld CA, Sawaya MR, Brahmandam V, Cascio D, Ho KK, Trevithick-Sutton CC, Krogmann DW, Yeates TO: The crystal structure of a cyanobacterial water-soluble carotenoid binding protein. Structure 2003, 11:55-65.

This work reported the first crystal structure of the OCP, demonstrated it to be an effective quencher of ${ }^{1} \mathrm{O}_{2}$, discovered its structural domains could be encoded as separate genes, noted its ability to change color to red, suggested it would be suitable for a photoprotective function.

7. Sedoud A, Lopez-Igual R, Rehman AU, Wilson A, Perreau F, Boulay C, Vass I, KriegerLiszkay A, Kirilovsky D: The Cyanobacterial Photoactive Orange Carotenoid Protein Is an Excellent Singlet Oxygen Quencher. Plant Cell 2014, 26:1781-1791.

8. Kerfeld CA: Structure and function of the water-soluble carotenoid-binding proteins of cyanobacteria. Photosynth Res 2004, 81:215-225.

9. $\bullet$ Melnicki MR, Leverenz RL, Sutter M, Lopez-Igual R, Wilson A, Pawlowski EG, Perreau F, Kirilovsky D, Kerfeld CA: Structure, Diversity, and Evolution of a New Family of Soluble Carotenoid-Binding Proteins in Cyanobacteria. Mol Plant 2016, 9:1379-1394. This work identified and classified OCP, FRP, HCP, and CTDH genes co-occurring among cyanobacterial genomes, phylogenomically sub-classified OCP-NTD homologs into distinct paralogous HCP sub-clades, revealed divergent expression patterns among HCP subtypes, and introduced a hypothesis for OCP evolution involving ancient domain fusion of a CTDH with an HCP4-like ancestor. The first crystal structures of an HCP are also presented, using HCP1 from Nostoc 7120 , demonstrating flexible binding of a range of carotenoids.

10. • Lopez-Igual R, Wilson A, Leverenz RL, Melnicki MR, Bourcier de Carbon C, Sutter M, Turmo A, Perreau F, Kerfeld CA, Kirilovsky D: Different Functions of the Paralogs to the N-Terminal Domain of the Orange Carotenoid Protein in the Cyanobacterium Anabaena sp. PCC 7120. Plant Physiol 2016, 171:1852-1866. 
This paper confirmed carotenoid binding in four different HCPs from a heterocystous cyanobacterium, and revealed distinct functional properties for HCP4 (capable of constitutive PBS quenching) and for HCP2 and HCP3 (as efficient ${ }^{1} \mathrm{O}_{2}$ quenchers). Native HCP expression and sub-cellular localization patterns were examined and supported evidence for subfunctionalization.

11. Wilson A, Kinney JN, Zwart PH, Punginelli C, D'Haene S, Perreau F, Klein MG, Kirilovsky D, Kerfeld CA: Structural determinants underlying photoprotection in the photoactive orange carotenoid protein of cyanobacteria. J Biol Chem 2010, 285:18364-18375.

12. Wilson A, Punginelli C, Gall A, Bonetti C, Alexandre M, Routaboul JM, Kerfeld CA, van Grondelle R, Robert B, Kennis JT, et al.: A photoactive carotenoid protein acting as light intensity sensor. Proc Natl Acad Sci U S A 2008, 105:12075-12080.

13. Bourcier de Carbon C, Thurotte A, Wilson A, Perreau F, Kirilovsky D: Biosynthesis of soluble carotenoid holoproteins in Escherichia coli. Sci Rep 2015, 5:9085.

14. Mori Y: Computational study on the color change of $3^{\prime}$-hydroxyechinenone in the orange carotenoid protein. Chemical Physics Letters 2016, 652:184-189.

15. Wilson A, Punginelli C, Couturier M, Perreau F, Kirilovsky D: Essential role of two tyrosines and two tryptophans on the photoprotection activity of the Orange Carotenoid Protein. Biochim Biophys Acta 2011, 1807:293-301.

16. Punginelli C, Wilson A, Routaboul JM, Kirilovsky D: Influence of zeaxanthin and echinenone binding on the activity of the orange carotenoid protein. Biochim Biophys Acta 2009, 1787:280-288.

17. Zhang H, Liu H, Niedzwiedzki DM, Prado M, Jiang J, Gross ML, Blankenship RE: Molecular mechanism of photoactivation and structural location of the cyanobacterial orange carotenoid protein. Biochemistry 2014, 53:13-19.

18. - Gupta S, Guttman M, Leverenz RL, Zhumadilova K, Pawlowski EG, Petzold CJ, Lee KK, Ralston CY, Kerfeld CA: Local and global structural drivers for the photoactivation of the orange carotenoid protein. Proc Natl Acad Sci U S A 2015, 112:E5567-5574.

This paper used a combination of solution-state methods (MS-based X-ray footprinting, CD, and SAXS) to study OCP photoactivation in the absence of OCP ${ }^{\mathrm{R}}$ crystals, and identified both global and local structural changes upon photoactivation, including complete domain dissociation, unfolding of the N-terminal extension and its dissociation from the CTD, and rearrangement of the $\mathrm{H}$-bonding network associated with conserved residues and structurally-relevant water molecules.

19. Leverenz RL, Jallet D, Li MD, Mathies RA, Kirilovsky D, Kerfeld CA: Structural and functional modularity of the orange carotenoid protein: distinct roles for the $\mathrm{N}$ and C-terminal domains in cyanobacterial photoprotection. Plant Cell 2014, 26:426437.

20. $\bullet$ Leverenz RL, Sutter M, Wilson A, Gupta S, Thurotte A, Bourcier de Carbon C, Petzold CJ, Ralston C, Perreau F, Kirilovsky D, et al.: PHOTOSYNTHESIS. A 12 A carotenoid translocation in a photoswitch associated with cyanobacterial photoprotection. Science 2015, 348:1463-1466.

The crystal structure of the isolated OCP-NTD from Synechocystis revealed a novel carotenoid configuration associated with $O C P^{R}$-like optical and quenching properties, indicating that photoactivation of full-length OCP is accompanied by a $12 \AA$ translocation of the carotenoid molecule further into the NTD.

21. Wu YP, Krogmann DW: The orange carotenoid protein of Synechocystis PCC 6803. Biochim Biophys Acta 1997, 1322:1-7. 
22. Fraikin GY, Strakhovskaya MG, Belenikina NS, Rubin AB: Bacterial photosensory proteins: Regulatory functions and optogenetic applications. Microbiology 2015, 84:461-472.

23. - Liu H, Zhang H, Orf GS, Lu Y, Jiang J, King JD, Wolf NR, Gross ML, Blankenship RE: Dramatic Domain Rearrangements of the Cyanobacterial Orange Carotenoid Protein upon Photoactivation. Biochemistry 2016, 55:1003-1009.

Using isotopically-coded cross-linking and MS, this paper indicated that the relative position and orientation of the OCP-NTD and CTD dramatically change upon upon photoactivation, and used $O C P^{R}$-specific crosslinked pairs to constrain models of the domain dissociation, proposing that the CTD flips outward along an intrinsically unstructured loop which winds along the surface of the CTD in OCP

24. Maksimov EG, Shirshin EA, Sluchanko NN, Zlenko DV, Parshina EY, Tsoraev GV, Klementiev KE, Budylin GS, Schmitt FJ, Friedrich T, et al.: The Signaling State of Orange Carotenoid Protein. Biophysical Journal 2015, 109:595-607.

25. Liu H, Zhang $H$, King j D, Wolf NR, Prado M, Gross ML, Blankenship RE: Mass spectrometry footprinting reveals the structural rearrangements of cyanobacterial orange carotenoid protein upon light activation. Biochim Biophys Acta 2014, 1837:1955-1963.

26. Thurotte A, Lopez-Igual R, Wilson A, Comolet L, Bourcier de Carbon C, Xiao F, Kirilovsky D: Regulation of Orange Carotenoid Protein Activity in Cyanobacterial Photoprotection. Plant Physiology 2015, 169:737-747.

27. De Re E, Schlau-Cohen GS, Leverenz RL, Huxter VM, Oliver TA, Mathies RA, Fleming GR: Insights into the structural changes occurring upon photoconversion in the orange carotenoid protein from broadband two-dimensional electronic spectroscopy. J Phys Chem B 2014, 118:5382-5389.

28. Maksimov EG, Moldenhauer M, Shirshin EA, Parshina EA, Sluchanko NN, Klementiev KE, Tsoraev GV, Tavraz NN, Willoweit M, Schmitt F-J, et al.: A comparative study of three signaling forms of the orange carotenoid protein. Photosynthesis Research 2016, 130:389-401.

29. Zhang H, Liu H, Lu Y, Wolf NR, Gross ML, Blankenship RE: Native mass spectrometry and ion mobility characterize the orange carotenoid protein functional domains. Biochim Biophys Acta 2016, 1857:734-739.

30. Cogdell RJ, Gardiner AT: Activated OCP unlocks nonphotochemical quenching in cyanobacteria. Proc Natl Acad Sci U S A 2015, 112:12547-12548.

31. Boulay C, Wilson A, D'Haene S, Kirilovsky D: Identification of a protein required for recovery of full antenna capacity in OCP-related photoprotective mechanism in cyanobacteria. Proc Natl Acad Sci U S A 2010, 107:11620-11625.

32. Gwizdala M, Wilson A, Omairi-Nasser A, Kirilovsky D: Characterization of the Synechocystis PCC 6803 Fluorescence Recovery Protein involved in photoprotection. Biochim Biophys Acta 2013, 1827:348-354.

33. • Sutter M, Wilson A, Leverenz RL, Lopez-Igual R, Thurotte A, Salmeen AE, Kirilovsky D, Kerfeld CA: Crystal structure of the FRP and identification of the active site for modulation of OCP-mediated photoprotection in cyanobacteria. Proc Natl Acad Sci U S A 2013, 110:10022-10027.

This work determined the first FRP crystal structure, observing two different oligomeric configurations within the same asymmetric unit, each with a different fold topology within the respective monomers. Bioinformatics and structural analysis suggested that the dimer is the active form and functional and biochemical analyses of site-directed mutants identified critical residues as well as a possible active site. 
34. Lu Y, Liu H, Saer R, Zhang H, Meyer C, Li VL, Shi L, King JD, Gross ML, Blankenship RE: Native mass spectrometry analysis of oligomerization states of FRP and OCP: Two proteins involved in the cyanobacterial photoprotection cycle. Biochemistry 2016.

35. Sluchanko NN, Klementiev KE, Shirshin EA, Tsoraev GV, Friedrich T, Maksimov EG: The purple Trp288Ala mutant of Synechocystis OCP persistently quenches phycobilisome fluorescence and tightly interacts with FRP. Biochimica et Biophysica Acta (BBA) - Bioenergetics 2017, 1858:1-11.

36. Bonente G, Howes BD, Caffarri S, Smulevich G, Bassi R: Interactions between the Photosystem II Subunit PsbS and Xanthophylls Studied in Vivo and in Vitro. Journal of Biological Chemistry 2008, 283:8434-8445.

37. Li X-P, Gilmore AM, Caffarri S, Bassi R, Golan T, Kramer D, Niyogi KK: Regulation of Photosynthetic Light Harvesting Involves Intrathylakoid Lumen pH Sensing by the PsbS Protein. Journal of Biological Chemistry 2004, 279:22866-22874.

38. Fan M, Li M, Liu Z, Cao P, Pan X, Zhang H, Zhao X, Zhang J, Chang W: Crystal structures of the PsbS protein essential for photoprotection in plants. Nat Struct Mol Biol 2015, 22:729-735.

39. Gwizdala M, Wilson A, Kirilovsky D: In vitro reconstitution of the cyanobacterial photoprotective mechanism mediated by the Orange Carotenoid Protein in Synechocystis PCC 6803. Plant Cell 2011, 23:2631-2643.

40. Wilson A, Gwizdala M, Mezzetti A, Alexandre M, Kerfeld CA, Kirilovsky D: The essential role of the $\mathrm{N}$-terminal domain of the orange carotenoid protein in cyanobacterial photoprotection: importance of a positive charge for phycobilisome binding. Plant Cell 2012, 24:1972-1983.

41. $\bullet$ Harris D, Tal O, Jallet D, Wilson A, Kirilovsky D, Adir N: Orange carotenoid protein burrows into the phycobilisome to provide photoprotection. Proceedings of the National Academy of Sciences of the United States of America 2016, 113:E1655-E1662.

MS analysis of cross-linked OCP-PBS complexes was used to propose that the OCP-NTD burrows tightly into the PBS, buried in the interface between the external ApcD-containing APC trimer and the ApcEF-containing APC trimer, while the CTD is mobile and may sit on the exterior of the PBS core.

42. Elanskaya IV, Kononova IA, Lukashev EP, Bolychevtseva YV, Yanushin MF, Stadnichuk IN: Functions of chromophore-containing domain in the large linker LCM-polypeptide of phycobilisome. Doklady Biochemistry and Biophysics 2016, 471:403-406.

43. Jallet D, Thurotte A, Leverenz RL, Perreau F, Kerfeld CA, Kirilovsky D: Specificity of the cyanobacterial orange carotenoid protein: influences of orange carotenoid protein and phycobilisome structures. Plant Physiol 2014, 164:790-804.

44. Zhang H, Cui W, Gross ML, Blankenship RE: Native mass spectrometry of photosynthetic pigment-protein complexes. FEBS Lett 2013, 587:1012-1020.

45. Tian L, van Stokkum IH, Koehorst RB, Jongerius A, Kirilovsky D, van Amerongen H: Site, rate, and mechanism of photoprotective quenching in cyanobacteria. J Am Chem Soc 2011, 133:18304-18311.

46. Tian L, van Stokkum IH, Koehorst RB, van Amerongen $\mathrm{H}$ : Light harvesting and bluegreen light induced non-photochemical quenching in two different $\mathrm{C}$-phycocyanin mutants of Synechocystis PCC 6803. J Phys Chem B 2013, 117:11000-11006.

47. Kuzminov FI, Karapetyan NV, Rakhimberdieva MG, Elanskaya IV, Gorbunov MY, Fadeev VV: Investigation of OCP-triggered dissipation of excitation energy in PSI/PSII-less Synechocystis sp. PCC 6803 mutant using non-linear laser fluorimetry. Biochim Biophys Acta 2012, 1817:1012-1021. 
48. Kuzminov FI, Bolychevtseva YV, Elanskaya IV, Karapetyan NV: Effect of APCD and APCF subunits depletion on phycobilisome fluorescence of the cyanobacterium Synechocystis PCC 6803. J Photochem Photobiol B 2014, 133:153-160.

49. Bernat G, Schreiber U, Sendtko E, Stadnichuk IN, Rexroth S, Rogner M, Koenig F: Unique properties vs. common themes: the atypical cyanobacterium Gloeobacter violaceus PCC 7421 is capable of state transitions and blue-light-induced fluorescence quenching. Plant Cell Physiol 2012, 53:528-542.

50. Berera R, van Stokkum IH, Gwizdala M, Wilson A, Kirilovsky D, van Grondelle R: The photophysics of the orange carotenoid protein, a light-powered molecular switch. J Phys Chem B 2012, 116:2568-2574.

51. Maksimov EG, Schmitt FJ, Shirshin EA, Svirin MD, Elanskaya IV, Friedrich T, Fadeev VV, Paschenko VZ, Rubin AB: The time course of non-photochemical quenching in phycobilisomes of Synechocystis sp. PCC6803 as revealed by picosecond timeresolved fluorimetry. Biochim Biophys Acta 2014, 1837:1540-1547.

52. Berera R, Gwizdala M, van Stokkum IH, Kirilovsky D, van Grondelle R: Excited states of the inactive and active forms of the orange carotenoid protein. J Phys Chem $B$ 2013, 117:9121-9128.

53. Polivka T, Chabera $P$, Kerfeld CA: Carotenoid-protein interaction alters the $\mathbf{S}(1)$ energy of hydroxyechinenone in the Orange Carotenoid Protein. Biochim Biophys Acta 2013, 1827:248-254.

54. Wang Q, Moerner WE: Dissecting pigment architecture of individual photosynthetic antenna complexes in solution. Proc Natl Acad Sci U S A 2015, 112:13880-13885.

55. Schmidt D, Cho YK: Natural photoreceptors and their application to synthetic biology. Trends in Biotechnology 2015, 33:80-91.

56. Shcherbakova DM, Shemetov AA, Kaberniuk AA, Verkhusha VV: Natural Photoreceptors as a Source of Fluorescent Proteins, Biosensors, and Optogenetic Tools. Annual Review of Biochemistry 2015, 84:519-550.

57. - Kromdijk J, Glowacka K, Leonelli L, Gabilly ST, Iwai M, Niyogi KK, Long SP: Improving photosynthesis and crop productivity by accelerating recovery from photoprotection. Science 2016, 354:857-861.

This work demonstrated that tobacco plants engineered to have an accelerated xanthophyll cycle and increased PsbS were able to produce more biomass in natural field conditions, suggesting that the photosynthetic yield can be enhanced by speeding up the recovery steps of a photoprotective NPQ process.

58. Branco Dos Santos F, Du W, Hellingwerf KJ: Synechocystis: not just a plug-bug for CO2, but a green E. coli. Frontiers in Bioengineering and Biotechnology 2014, 2. 